\title{
Proximity effect in ferromagnet/superconductor hybrids: from diffusive to ballistic motion
}

\author{
Jacob Linder, ${ }^{1}$ Malek Zareyan, ${ }^{2}$ and Asle Sudb $\emptyset^{1}$ \\ ${ }^{1}$ Department of Physics, Norwegian University of Science and Technology, N-7491 Trondheim, Norway \\ ${ }^{2}$ Institute for Advanced Studies in Basic Sciences, 45195-1159, Zanjan, Iran
}

(Dated: Received November 23, 2018)

\begin{abstract}
We present an analytical study of the proximity effect in ferromagnet/superconductor $(\mathrm{F} / \mathrm{S})$ heterostructures, allowing for an arbitrary magnetic exchange energy as well as arbitrary impurity and spin-flip scattering rates within a quasiclassical approach. While previous studies mainly have focused on the clean or dirty limits, our results grant access to the regime of intermediate impurity concentrations, thus allowing us to probe the crossover from the clean to dirty limit. We find that in the crossover regime, all possible symmetry correlations of the proximity-induced anomalous Green's function are induced in the ferromagnet. We also point out that the local density of states oscillates spatially, not only for an F/S bilayer, but also for a normal/superconductor (N/S) bilayer in the diffusive limit, a fact which appears to have gone unnoticed in the literature. Within the weak-proximity effect regime, we present compact analytical expressions valid for arbitrary exchange fields and impurity scattering rates for $i$ ) the local density of states in an F/S bilayer, ii) the Josephson current in an $\mathrm{S} / \mathrm{F} / \mathrm{S}$ junction, and iii) the critical temperature in an F/S/F multilayer. For all cases, we study in particular the crossover regime between diffusive and ballistic motion. Our results may be useful for analyzing experimental data in cases when the dirty limit is not fully reached, thus invalidating the use of the Usadel equation.
\end{abstract}

PACS numbers: 74.25.Fy,74.45.+c,74.50.+r,74.62.-c

\section{INTRODUCTION}

The interest in ferromagnet/superconductor $(\mathrm{F} / \mathrm{S})$ heterostructures has increased much during the last decade $e^{1.2 .3}$. This may probably be attributed to advances in experimental fabrication/deposition techniques as well as intriguing theoretical predictions. The main hope is that future devices and applications will rely on manipulation of not only the electron charge but also its spin. Based on this idea, a new research area known as superspintronics has emerged, aiming at utilization of charge and spin transport in ferromagnet/superconductor heterostructures. For instance, several authors have investigated the possibility of dissipationless currents of spin and charge in magnetically ordered superconductors $4,5,6,7,8,9,10,11$. A large number of other studies related to spin degrees of freedom in superconducting systems has also appeared in the literature ${ }^{12,13,14,15}$.

A considerable amount of attention has been devoted to the arguably most simple experimental laboratory where the interplay between ferromagnetism and superconductivity may be studied, namely a F/S bilayer. The two long-range order phenomena mix close to the interface, giving rise to interesting effects both from a basic physics perspective and in terms of potential applications. These effects include induction of unusual superconducting symmetry correlations and a highly non-monotonic behaviour of various physical quantities on the size of the system. The latter is a result of the non-uniform superconducting correlations that are induced in the ferromagnetic layer by means of the proximity effect.

As a natural extension of the F/S bilayer, there has also been much focus on $\mathrm{S} / \mathrm{F} / \mathrm{S}$ systems and F/S/F systems, where the influence of ferromagnetism on the Josephson current and the critical temperature has been studied, respectively. The large majority of works related to these systems assumed that the diffusive limit was reached. In this case, elastic scattering on impurities renders the Green's function to be isotropic in space, while it may still retain a complicated spin-structure. From an experimental point of view, the diffusive regime is certainly relevant, but there are nevertheless some complications. One point bears upon the theoretical framework used to study the physics in the diffusive regime. The quasiclassical Usade ${ }^{16}$ equation is widely employed to study the proximity effect in F/S heterostructures, and is valid under two main assumptions. Firstly, that the Fermi energy is much larger than any other energy scale and the essential physics is governed by fermions at Fermi level, and secondly, that the inverse impurity scattering rate is much larger than any other energy scale except for the Fermi energy. For strong ferromagnets such as $\mathrm{Co}$ or $\mathrm{Ni}$, the second condition may be violated. In that case, one must revert to the more general Eilenberger ${ }^{17}$ equation, which is only subject to the first condition.

The Eilenberger equation is more complicated to solve analytically than the Usadel equation, although some special limits permit fairly simple analytical expressions. Let $h$ denote the exchange-energy of the ferromagnet while $\tau_{\text {imp }}$ denotes the inverse impurity scattering rate. The Usadel equation is then obtained from the Eilenberger equation by demanding $h \tau_{\text {imp }} \ll 1$, while the case of a strong and clean ferromagnet is obtained in the limit $h \tau_{\text {imp }} \gg 1$. We assume that $h \gg \Delta$ is fulfilled. In Ref $\stackrel{18}{=}$, some aspects of the DOS in F/S heterostructures were considered to leading order in the parameter $\left(h \tau_{\mathrm{imp}}\right)^{-1}$, corresponding to a strong ferromagnet which falls outside the range of applicability of the Usadel equation. In Ref $\frac{19}{\underline{1}}$, the Josephson current in an $\mathrm{S} / \mathrm{F} / \mathrm{S}$ structure was also investigated for the case of a strong ferromagnet, $h \tau_{\text {imp }} \gg 1$. Some authors have also considered F/S heterostructures where the impurity scattering rate was disregarded or assumed to be small, corresponding to the ballistic regime. $20,21,22,23,24,25,26,27$

Although the agreement between theory and experiment in this research area has proven to be satisfactory in many cases, there are still discrepancies to be accounted for. For instance, the Usadel equation has failed to account quantitatively for 
the critical temperature in $\mathrm{F} / \mathrm{S} / \mathrm{F}$ spin-valves. Furthermore, anomalous features in the DOS for a very thin F/S bilayer that could not be accounted for even qualitatively, were reported in Ref $\stackrel{28}{2}$. Moreover, the Usadel equation approach fails from the start when addressing systems with strong ferromagnets.

All of this points to the need of taking the role of impurity scattering more seriously. In this paper, we aim at doing precisely so by solving the Eilenberger equation analytically and studying the crossover regime between ballistic and diffusive motion (see Fig. 1). To illustrate how various physical quantities behave in this crossover regime, we study i) the local density of states in an F/S bilayer, $i i$ ) the Josephson current in an $\mathrm{S} / \mathrm{F} / \mathrm{S}$ junction, and iii) the critical temperature in an $\mathrm{F} / \mathrm{S} / \mathrm{F}$ multilayer for arbitrary values of $h$ and $\tau$ (within the quasiclassical approach). In each case, we present compact analytical formula to facilitate comparison to experimental data in cases where the diffusive limit may not be fully warranted or where strong ferromagnets are involved.

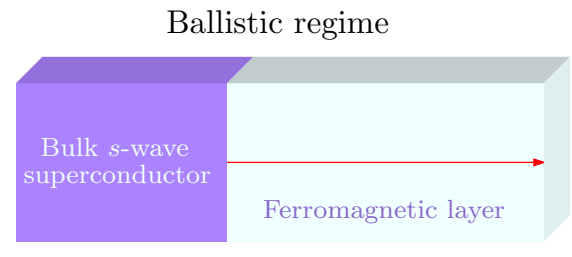

Intermediate regime

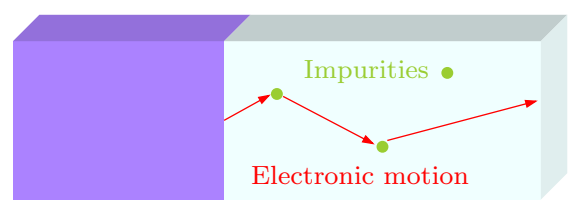

Diffusive regime

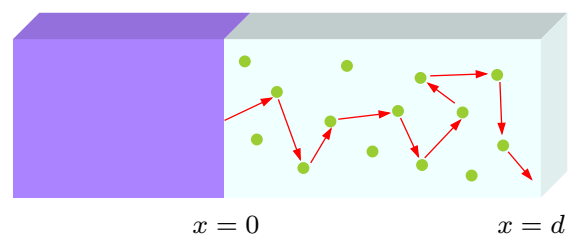

FIG. 1: (color online) Overview of the superconductor/ferromagnet heterostructure we will study in this paper. We take into account an arbitrary strength of the exchange field as well as an arbitrary rate of non-magnetic and magnetic scattering within a quasiclassical approach.

This paper is organized as follows. In Sec. II, we establish the theoretical framework which is employed in this work. In Sec. III] we present our main results with belonging discussion: the DOS of an F/S bilayer in Sec. III B, the Josephson current in an S/F/S multilayer in Sec. IIIC, and finally the critical temperature in an F/S/F multilayer in Sec. [IID Eqs. (25), (32), and (37) are the main analytical results of this work. We conclude in Sec. IV] Below, we will use boldface notation for vectors, $\ldots$ for $2 \times 2$ matrices, and . . . for $4 \times 4$ matrices. The reader may consult the Appendix for a definition of the generalized Pauli-matrices we employ in this paper.

\section{THEORETICAL FORMULATION}

The Eilenberger equation reads ${ }^{17}$

$$
1 \mathbf{v}_{F} \cdot \nabla \hat{g}+\left[\varepsilon \hat{\rho}_{3}+\hat{M}-\hat{V}_{\text {imp }}-\hat{S}_{\text {flip }}+\hat{\Delta}, \hat{g}\right]=0,
$$

where $\hat{g} \equiv \hat{g}^{\mathrm{R}}\left(\mathbf{R}, \varepsilon, \mathbf{p}_{F}\right)$ is the retarded part of the Green's function. Here, $\varepsilon$ is the quasiparticle energy, $\mathbf{R}$ is the centerof-mass coordinate, and $\mathbf{p}_{F}\left(\mathbf{v}_{F}\right)$ is the Fermi momentum (velocity) vector. The self-energies that enter Eq. (1) are the magnetic exchange energy $\hat{M}=h \operatorname{diag}\left\{\underline{\tau_{3}}, \underline{\tau_{3}}\right\}$, the impurity scattering $\hat{V}_{\text {imp }}=-\left[1 /\left(2 \tau_{\text {imp }}\right)\right]\langle\hat{g}\rangle$, the (uniaxial) spin-flip scattering $\hat{S}_{\text {flip }}=-\left[1 /\left(2 \tau_{\text {flip }}\right)\right] \hat{\rho}_{3}\langle\hat{g}\rangle \hat{\rho}_{3}$, and the superconducting order parameter

$$
\hat{\Delta}=\left(\begin{array}{cc}
\underline{0} & 1 \tau_{2} \Delta_{2} \Delta \\
{ }_{1}{ }^{*}
\end{array}\right) .
$$

All matrices used above $\left(\hat{\rho}_{i}, \underline{\tau_{i}}\right)$ are defined in the Appendix [Eq. (41)]. The brackets $\langle\ldots\rangle$ denote an angular average over the Fermi surface. Also, $h$ is the exchange splitting while $\tau_{\text {imp(flip) }}$ is the scattering time associated with impurity (spinflip) scattering. We may conveniently rewrite Eq. (1) as:

$$
\begin{aligned}
1 \mathbf{v}_{F} \cdot \nabla & \underline{g_{\sigma}}+\left[(\varepsilon+\sigma h) \underline{\tau_{3}}+\sigma \underline{\Delta}+\frac{1}{2 \tau_{\mathrm{imp}}}\left\langle\underline{g_{\sigma}}\right\rangle\right. \\
& \left.+\frac{1}{2 \tau_{\mathrm{sf}}} \underline{\tau_{3}}\left\langle\underline{g_{\sigma}}\right\rangle \underline{\tau_{3}}, \underline{g_{\sigma}}\right]=0, \sigma=\uparrow, \downarrow= \pm 1
\end{aligned}
$$

where the superconducting order parameter matrix $\underline{\Delta}$ reads

$$
\underline{\Delta}=\left(\begin{array}{cc}
0 & \Delta \\
-\Delta^{*} & 0
\end{array}\right), \Delta=\Delta_{0} \mathrm{e}^{1 \chi},
$$

upon letting $\chi$ denote the phase corresponding to the globally broken $\mathrm{U}(1)$ symmetry in the superconducting state. The brackets $\langle\ldots\rangle$ denote angular averaging over the Fermi surface. We employ the Ricatti parametrization ${ }^{29}$ of the Green's function:

$$
\underline{g_{\sigma}}=\mathcal{N}_{\sigma}\left(\begin{array}{cc}
1-a_{\sigma} b_{\sigma} & 2 a_{\sigma} \\
2 b_{\sigma} & -1+a_{\sigma} b_{\sigma}
\end{array}\right), \mathcal{N}_{\sigma}=\left(1+a_{\sigma} b_{\sigma}\right)^{-1} .
$$

Here, $a_{\sigma}$ and $b_{\sigma}$ are two unknown functions used to parametrize the Green's functions. They will be determined by solving the Eilenberger equation with appropriate boundary conditions. A general treatment of the Eilenberger equation calls for a numerical solution. In the case of a weak proximity effect, however, the Eilenberger equation may be linearized in the anomalous part of the Green's function which permits an analytical approach. The assumption of a weak proximity effect corresponds mathematically to a scenario where higher order terms of $\left\{a_{\sigma}, b_{\sigma}\right\}$ are disregarded in the Eilenberger equation, i.e. one assumes that $\left|a_{\sigma}\right| \ll 1,\left|b_{\sigma}\right| \ll$ 1. In an experimental situation, a weak proximity effect in F/S heterostructures may be expected whenever the tunneling limit is reached and the number of conducting channels at the interface is low. Also, assuming a superconducting reservoir, the proximity effect becomes weaker in magnitude upon increasing the thickness of the ferromagnetic layer. 
The spatial depletion of the superconducting order parameter near the S/F interface will be disregarded. This is an excellent approximation in the corresponding low-transparency regime, which will be considered throughout this paper except for in Sec. IIID where this issue is discussed further. At the S/F interface $(x=0)$ we use Zaitsev's boundary conditions. Define the symmetric and antisymmetric part of the Green's function as

$$
\left.\underline{\mathcal{S}_{\sigma, i}}=\frac{1}{2}{\underline{g_{\sigma, i}}}^{+}+\underline{g_{\sigma, i}}{ }^{-}\right), \underline{\mathcal{A}_{\sigma, i}}=\frac{1}{2}\left({\underline{g_{\sigma, i}}}^{+}-{\underline{g_{\sigma, i}}}^{-}\right),
$$

where the \pm superscript on the Green's function denotes right/left-going quasiparticle excitations and the subscript $i$ denotes the ferromagnetic or superconducting region. The first of Zaitsev's boundary conditions ${ }^{30}$ demands continuity of the antisymmetric part $\mathcal{A}_{\sigma, i}$ of the Green's function. The second one relates the Green's functions in the ferromagnetic and superconducting regions to the interface transparency. We obtain

$$
\begin{aligned}
{\underline{\mathcal{A}_{\sigma, F}}}\left[\mathcal{R}\left(1-{\underline{\mathcal{A}_{\sigma, F}}}^{2}\right)\right. & \left.+\frac{\mathcal{T}}{4}\left(\underline{\mathcal{S}_{\sigma, S}}-\underline{\mathcal{S}_{\sigma, F}}\right)^{2}\right] \\
& =\frac{\mathcal{T}}{4}\left[\underline{\mathcal{S}_{\sigma, F}}, \underline{\mathcal{S}_{\sigma, S}}\right]_{-},
\end{aligned}
$$

where $\mathcal{R}$ and $\mathcal{T}$ are the reflection and transmission coefficients satisfying $\mathcal{R}+\mathcal{T}=1$, and $[\ldots]_{-}$denotes a commutator. High and low transparency interfaces correspond to $\mathcal{T} \simeq 1$ and $\mathcal{T} \ll 1$, respectively. Although Eq. (6) is expressed rather compactly, a general solution for arbitrary $\mathcal{T}$ and $\mathcal{R}$ is very hard to obtain. In the experimentally relevant situation, one may assume that $\mathcal{T} \ll \mathcal{R}$. For a low-transparency barrier and a weak proximity effect, Eq. (6) simplifies greatly to

$$
\left.\underline{\mathcal{A}_{\sigma, F}}\right|_{x=0}=\left.\gamma\left[\mathcal{S}_{\sigma, F}, \mathcal{S}_{\sigma, S}\right]_{-}\right|_{x=0},
$$

where $\gamma=\mathcal{T} /(4 \mathcal{R})$ is a measure of the barrier transparency. At the end of the ferromagnetic layer, we demand $\left.\underline{\mathcal{A}_{\sigma, F}}\right|_{x=d}=\underline{0}$.

We consider here an effective one-dimensional calculation, which should provide sound results due to the isotropic nature of the ferromagnetic and superconducting order parameters. We do not expect any qualitative differences from a twodimensional or three-dimensional model, since the superconducting gap and the magnetic exchange field do not depend on the quasiparticle momenta, and since there are no surfacebound states ${ }^{31}$ at the interfaces of the systems we consider. Thus, it should be possible to capture the essential physics by studying an effective one-dimensional model, which permits us to proceed analytically. This point of view is supported by the fact that, as seen later in this work, we reproduce in limiting cases previous results obtained in the literature which employed a two-dimensional calculation.

Under the assumption of a weak proximity effect, the Eilen- berger equations in the ferromagnetic region take the form:

$$
\begin{aligned}
\alpha 1 v_{F} \partial_{x} a_{\sigma}+2 a_{\sigma}(\varepsilon+\sigma h) & +\frac{1}{2 \tau_{\mathrm{imp}}}\left(a_{\sigma}^{\alpha}-a_{\sigma}^{-\alpha}\right) \\
& +\frac{1}{2 \tau_{\mathrm{sf}}}\left(3 a_{\sigma}^{\alpha}+a_{\sigma}^{-\alpha}\right)=0 \\
\alpha 1 v_{F} \partial_{x} b_{\sigma}-2 b_{\sigma}(\varepsilon+\sigma h) & -\frac{1}{2 \tau_{\mathrm{imp}}}\left(b_{\sigma}^{\alpha}-b_{\sigma}^{-\alpha}\right) \\
& -\frac{1}{2 \tau_{\mathrm{sf}}}\left(3 b_{\sigma}^{\alpha}+b_{\sigma}^{-\alpha}\right)=0,
\end{aligned}
$$

where $\alpha= \pm$ denotes right- and left-going quasiparticles, respectively. It is necessary to take into account the direction of the quasiparticles at Fermi level due to the term $\mathbf{v}_{F} \cdot \nabla \hat{g}$ in Eq. (1). Thus, $\sigma$ denotes the spin direction while $\alpha$ denotes the direction of motion in $a_{\sigma}^{\alpha}$ and likewise for $b_{\sigma}^{\alpha}$. The impurity and spin-flip scattering self-energies enter Eq. (8) by means of the matrices $\hat{V}_{\text {imp }}$ and $\hat{S}_{\text {flip }}$ in Eq. (1), which both depend on the Fermi-surface averaged Green's function. For a weak proximity effect, we have

$$
\left\langle\underline{g_{\sigma}}\right\rangle=\left(\begin{array}{cc}
1 & a_{\sigma}^{+}+a_{\sigma}^{-} \\
b_{\sigma}^{+}+b_{\sigma}^{-} & -1
\end{array}\right) .
$$

For a bulk ferromagnet, the solution is $a_{\sigma}^{ \pm}=b_{\sigma}^{ \pm}=0$.

In Ref $\stackrel{18}{ }$, the DOS in a S/F bilayer was studied by neglectinb both spin-flip scattering $\left(\tau_{\text {sf }} \rightarrow \infty\right)$ and the coupling term between the right- and left-going excitations in Eq. (8). In this case, one finds that Eq. (8) reduces to

$$
\begin{aligned}
& \pm 1 v_{F} \partial_{x} a_{\sigma}^{ \pm}+\left[2(\varepsilon+\sigma h)+\frac{1}{2 \tau_{\mathrm{imp}}}\right] a_{\sigma}^{ \pm}=0, \\
& \pm 1 v_{F} \partial_{x} b_{\sigma}^{ \pm}-\left[2(\varepsilon+\sigma h)+\frac{1}{2 \tau_{\mathrm{imp}}}\right] b_{\sigma}^{ \pm}=0 .
\end{aligned}
$$

The decaying solution for $x \rightarrow \infty$ of the above equations reads

$$
\begin{array}{r}
a_{\sigma}^{+}=k_{a \sigma} \exp \left[-\kappa_{\sigma} x / l\right], b_{\sigma}^{-}=k_{b \sigma} \exp [-\kappa x / l], \\
\kappa_{\sigma}=1-21(\varepsilon+\sigma h) \tau_{\text {imp }}, l=v_{F} \tau_{\text {imp }} .
\end{array}
$$

while $a_{\sigma}^{-}=b_{\sigma}^{+}=0$. Above, $k_{a \sigma}$ and $k_{b \sigma}$ are constants to be determined from the boundary condition at $x=0$, and the structure of the Green's function becomes

$$
\mathcal{S}_{\sigma, F}=\left(\begin{array}{cc}
1 & a_{\sigma}^{+} \\
b_{\sigma}^{-} & -1
\end{array}\right), \mathcal{A}_{\sigma, F}=\left(\begin{array}{cc}
0 & a_{\sigma}^{+} \\
-b_{\sigma}^{-} & 0
\end{array}\right) .
$$

This shows how the decay length of the proximity-induced anomalous Green's function in the ferromagnet is governed by the mean free path $l$, and that it is independent of the exchange field in this main approximation. We now present a more rigorous solution by fully taking into account the coupling-term in Eqs. (8). To solve this problem, we note that Eqs. (8) may be written as a matrix differential equation:

$$
\begin{gathered}
\partial_{x} \mathbf{a}_{\sigma}=\underline{M_{a \sigma}} \mathbf{a}_{\sigma}, \mathbf{a}_{\sigma}=\left[a_{\sigma}^{+} a_{\sigma}^{-}\right]^{\mathrm{T}}, \\
\underline{M_{a \sigma}}=\frac{1}{v_{F}}\left(\begin{array}{cc}
r_{\sigma} & g \\
-g & -r_{\sigma}
\end{array}\right),
\end{gathered}
$$


where $\mathrm{T}$ denotes matrix transpose and we have defined the auxiliary quantities

$$
\begin{gathered}
r_{\sigma}=21(\varepsilon+\sigma h)-\left(g_{\mathrm{imp}}+3 g_{\mathrm{sf}}\right) / 2, \\
g=\left(g_{\mathrm{imp}}-g_{\mathrm{sf}}\right) / 2, g_{\mathrm{imp}(\mathrm{sf})} \equiv \tau_{\mathrm{imp}(\mathrm{sf})}^{-1}
\end{gathered}
$$

Diagonalizing $\underline{M_{\sigma}}$ according to $\underline{D_{\sigma}}=\underline{P}_{\sigma}{ }^{-1} \underline{M_{\sigma} P_{\sigma}}$, we obtain the trivial set of decoupled differential equations

$$
\partial_{x} \tilde{\mathbf{a}}_{\sigma}=\underline{D_{\sigma}} \tilde{\mathbf{a}}_{\sigma}, \tilde{\mathbf{a}}_{\sigma}=\underline{P}^{-1} \mathbf{a}_{\sigma} .
$$

From the above, we find that

$$
\tilde{a}_{\sigma}^{ \pm}=C_{a, \sigma}^{ \pm} \mathrm{e}^{ \pm \lambda_{\sigma} x}, \lambda_{\sigma}=v_{F}^{-1} \sqrt{r_{\sigma}^{2}-g^{2}},
$$

while the diagonalization matrix $\underline{P_{\sigma}}$ reads

$$
\begin{aligned}
\underline{P_{\sigma}} & =\left(\begin{array}{ll}
p_{1 \sigma} & p_{2 \sigma} \\
p_{2 \sigma} & p_{1 \sigma}
\end{array}\right), G_{\sigma}=g /\left(v_{F} \lambda_{\sigma}+r_{\sigma}\right), \\
p_{1 \sigma} & =N_{\sigma}, p_{2 \sigma}=-N_{\sigma} G_{\sigma}, N_{\sigma}=\left(1+\left|G_{\sigma}\right|^{2}\right)^{-1 / 2}
\end{aligned}
$$

In the superconducting region, we employ the bulk solution under the assumption that the interface transparency is low and that the ferromagnetic layer is much more disordered than the superconductor ${ }^{1}$. In this main approximation, we may employ the bulk solution of the Green's function in the superconductor:

$$
\underline{g_{\sigma}^{ \pm}}=\left(\begin{array}{cc}
c(\theta) & \sigma s(\theta) \\
-\sigma s(\theta) & -c(\theta)
\end{array}\right)
$$

with the definitions $c(\theta)=\cosh (\theta), s(\theta)=\sinh (\theta), \theta=$ $\operatorname{atanh}(\Delta / \varepsilon)$. Once the expression for the Green's function in the ferromagnet has been obtained, one may calculate various physical quantities of interest. By approximating $\mathcal{S}_{\sigma, F} \simeq \underline{\tau_{3}}$ in Eq. (7) in accordance with a weak proximity effect, we obtain for the case where the impurity-scattering coupling between the Ricatti-equations is ignored:

$$
\underline{g_{\sigma, F}^{ \pm}}=\underline{\tau_{3}}+2 \gamma \sigma s(\theta) \exp \left(-\kappa_{\sigma} x / l\right)\left(\underline{\tau_{1}} \pm \underline{1} \underline{\tau_{2}}\right)
$$

which is precisely the result of Ref ${ }^{18}$ for two semi-infinite superconducting and ferromagnetic layers in contact. When the coupling is properly taken into account, in addition to the vacuum boundary condition at $x=d$, we find that

$$
\begin{aligned}
\frac{g_{\sigma, F}^{ \pm}}{t} & =\left(\begin{array}{cc}
1 & 2 a_{\sigma}^{ \pm} \\
2 b_{\sigma}^{ \pm} & -1
\end{array}\right), \text { upon defining } \\
a_{\sigma}^{ \pm} & =p_{\sigma}^{ \pm} C_{1 \sigma}\left(\lambda_{\sigma}\right)+p_{\sigma}^{\mp} C_{2 \sigma}\left(\lambda_{\sigma}\right), \\
b_{\sigma}^{ \pm} & =p_{\sigma}^{ \pm} C_{1 \sigma}\left(-\lambda_{\sigma}\right)+p_{\sigma}^{\mp} C_{2 \sigma}\left(-\lambda_{\sigma}\right), \\
C_{1 \sigma} & =\frac{2 \gamma \sigma s(\theta) \mathrm{e}^{\lambda_{\sigma} x}}{p_{\sigma}^{+}-p_{\sigma}^{-}}\left[1-\frac{\mathrm{e}^{\lambda_{\sigma} d}}{2 \sinh \left(\lambda_{\sigma} d\right)}\right], \\
C_{2 \sigma} & =-\frac{\gamma \sigma s(\theta) \mathrm{e}^{\lambda_{\sigma}(d-x)}}{\left(p_{\sigma}^{+}-p_{\sigma}^{-}\right) \sinh \left(\lambda_{\sigma} d\right)}
\end{aligned}
$$

and $p_{\sigma}^{ \pm}=p_{1,2 \sigma}$. Note that in the diffusive limit where $g_{\text {imp }} \gg\left\{h, \varepsilon, \Delta_{0}, g_{\text {sf }}\right\}$, one would expect that the distinction between right-going and left-going particles is removed, such that ${\underline{g_{\sigma, F}}}^{+}=\underline{g}_{\sigma, F}{ }^{-}$. This is easily shown by exploiting

$$
\lim _{g_{\mathrm{imp}} \gg\left\{h, \varepsilon, \Delta_{0}, g_{\mathrm{sf}}\right\}}\left(v_{F} \lambda_{\sigma}+r_{\sigma}\right)=-g_{\mathrm{imp}} / 2,
$$

as seen from the previous equations. We also want to compare the results for $g_{\text {imp }} \gg\left\{h, \varepsilon, \Delta_{0}, g_{\text {sf }}\right\}$ with those obtained when using the linearized Usadel equation. The Usadel equation in a diffusive ferromagnet then reads

$$
D \partial_{x}^{2} f_{ \pm}+21\left(\varepsilon+1 g_{\mathrm{sf}} \pm h\right) f_{ \pm}=0
$$

where $f_{ \pm}=f_{t} \pm f_{s}$ and $f_{t}$ is the odd-frequency triplet anomalous Green's function while $f_{s}$ is the even-frequency singlet anomalous Green's function (both are isotropic in momentum space). We obtain that the only physically acceptable (decaying for $x \rightarrow \infty$ ) solution is

$$
\begin{gathered}
f_{+}=f_{0} \mathrm{e}^{1 k_{+} x} \text { if } \varepsilon>0, f_{-}=f_{0} \mathrm{e}^{-1 k_{-} x} \text { if } \varepsilon<0, \\
k_{ \pm}=\sqrt{21\left(\varepsilon+1 g_{\mathrm{sf}} \pm h\right) / D},
\end{gathered}
$$

where $f_{0}$ is a constant to be determined from the boundary conditions. Above, $D$ is the diffusion constant. For consistency, we should be able to obtain the same decaying solution from Eq. (19) when $g_{\text {imp }} \gg\left\{h, \varepsilon, \Delta_{0}, g_{\text {sf }}\right\}$. Focusing on the wavevector, we see that in this limit:

$$
\begin{aligned}
\lambda_{\sigma} & \rightarrow v_{F}^{-1} \sqrt{-21(\varepsilon+\sigma h) g_{\mathrm{imp}}+2 g_{\mathrm{imp}} g_{\mathrm{sf}}} \\
& =\sqrt{-21\left(\varepsilon+\sigma h+1 g_{\mathrm{sf}}\right) / D},
\end{aligned}
$$

where $D=v_{F}^{2} \tau_{\text {imp }}$ is the diffusion constant in one dimension (in three dimensions, $D=v_{F}^{2} \tau_{\text {imp }} / 3$ ). Eq. (23) is then consistent with the form of Eq. (22).

With a complete description of the behaviour of the Green's function in the ferromagnetic region, we now investigate the influence of the proximity effect on the local density of states (LDOS), and also study the singlet and triplet superconducting order parameters induced in the ferromagnet. The normalized LDOS as obtained from the solution of the Eilenberger equation may be written as

$$
N(\varepsilon, x)=\frac{1}{2} \sum_{\sigma}\left\langle\operatorname{Re}\left\{1+4 a_{\sigma}(\varepsilon, x) b_{\sigma}(\varepsilon, x)\right\}\right\rangle
$$

for a weak proximity effect. In the normal state, the normalized DOS is $N_{0}=1$. Inserting the expressions for $a_{\sigma}^{ \pm}$and $b_{\sigma}^{ \pm}$ into the above equation yields 


$$
N(x, \varepsilon)=1-\operatorname{Re}\left\{\sum_{\sigma} \frac{2 \gamma^{2} s^{2}(\theta)}{\sinh \left(\lambda_{\sigma} d\right)\left(1+G_{\sigma}\right)^{2}} \times\left[1+G_{\sigma}^{2}-2 G_{\sigma}\left(2 \sinh \left(\lambda_{\sigma} d-2 \lambda_{\sigma} x\right)+\frac{\cosh \left(2 \lambda_{\sigma} x\right)}{\sinh \left(\lambda_{\sigma} d\right)}\right)\right]\right\}
$$

Eq. (25) is the first of our three main analytical results in this work. Within the weak proximity effect regime, it provides a general expression for the DOS, taking into account an arbitrary exchange field and impurity scattering rate. As seen, the correction to the normal state DOS $N_{0}=1$ is zero for a vanishing interface transparency $(\gamma=0)$. While the weak proximity restriction only allows access to variations from the normal-state of DOS of around $10 \%$, this seems to be sufficient for the experimentally relevant situation. For instance, the deviation from the normal-state DOS due to the superconducting proximity effect was of order $1 \%$ in Ref. 32 .

In order to study the superconducting correlations inside the ferromagnetic region, first note that the full structure of the retarded Green's function is

$$
\hat{g}^{\mathrm{R}}=\left(\begin{array}{cc}
\underline{g} & \underline{f} \\
-\underline{\tilde{f}} & -\underline{\tilde{g}}
\end{array}\right),
$$

where the spin-structure reads

$$
\underline{f}=\left(\begin{array}{cc}
f_{\uparrow \uparrow} & f_{\uparrow \downarrow} \\
f_{\downarrow \uparrow} & f_{\downarrow \downarrow}
\end{array}\right)
$$

and we have defined $f_{\alpha \beta}=f_{\alpha \beta}\left(\mathbf{p}_{\mathrm{F}}, \varepsilon, x\right)$ and $\tilde{f}\left(\mathbf{p}_{\mathrm{F}}, \varepsilon, x\right)=$ $f\left(-\mathbf{p}_{\mathrm{F}},-\varepsilon, x\right)^{*}$. From the Ricatti-parametrization, we may define the different symmetry-components of the anomalous Green's functions as follows:

$$
\begin{aligned}
& f_{\mathrm{ESE}}=\sum_{\sigma} \sigma\left(a_{\sigma}^{+}+a_{\sigma}^{-}\right), f_{\mathrm{OSO}}=\sum_{\sigma} \sigma\left(a_{\sigma}^{+}-a_{\sigma}^{-}\right), \\
& f_{\mathrm{ETO}}=\sum_{\sigma}\left(a_{\sigma}^{+}-a_{\sigma}^{-}\right), f_{\mathrm{OTE}}=\sum_{\sigma}\left(a_{\sigma}^{+}+a_{\sigma}^{-}\right) .
\end{aligned}
$$

Here, the abbreviations are explained in Tab. I] Note that in the general case of finite $h$ and $\tau_{\text {imp }}$, all possible symmetry components of the anomalous Green's function are induced in the non-superconducting region. In the case of $h=0$, one may confirm from Eq. (19) that $a_{\sigma}^{ \pm} \rightarrow \sigma a^{ \pm}$, where $a^{ \pm}$ is independent of $\sigma$, such that $f_{\text {OTE }}=f_{\mathrm{ETO}}=0$. Physically, the induction of other symmetry components than $f_{\mathrm{ESE}}$, corresponding to the bulk superconductor, may be explained as follows ${ }^{33,34}$. In a normal metal/superconductor junction, the translational symmetry is broken at the interface separating the two regions. This causes even-parity and odd-parity components of the Green's function to mix near the interface. Since the Pauli-principle must be satisfied at all times, a change in the parity-symmetry of the Green's function must be accompanied by a change in either spin- or frequencysymmetry. In the absence of an exchange field, nothing breaks the spin symmetry, such that only the frequency-symmetry may be altered indirectly by the broken translational symmetry. However, if the spin-symmetry is also broken by replacing the normal metal with a ferromagnet, the spin-symmetry of the Green's function may also be altered. These considerations are summarized in Tab. I The possibility of a bulk oddfrequency superconducting state was discussed in Refs ${ }^{35.36}$, and there has very recently been made some predictions concerning characteristic transport properties of such a bulk oddfrequency superconducting state. ${ }^{33,37,38,39}$.

TABLE I: Proximity-induced anomalous Green's functions in a normal metal in contact with a conventional BCS-superconductor, which has an even-frequency spin-singlet even-parity symmetry. Below, the quasiballistic limit regime is characterized by a vanishing or small value of the impurity scattering rate, while the diffusive limit is characterized by an impurity scattering rate which dominates all other energy scales in the problem (except for the Fermi energy).

\begin{tabular}{ccccc}
\hline \hline Symmetry & $h \neq 0$, quasiballistic & $h=0$, quasiballistic & $h \neq 0$, diffusive & $h=0$, diffusive \\
\hline Even-frequency spin-singlet even-parity (ESE) & $\sqrt{ }$ & $\sqrt{ }$ & $\sqrt{ }$ & $\sqrt{ }$ \\
Odd-frequency spin-singlet odd-parity (OSO) & $\sqrt{ }$ & $\sqrt{ }$ & - & - \\
Even-frequency spin-triplet odd-parity (ETO) & $\sqrt{ }$ & - & - & - \\
Odd-frequency spin-triplet even-parity (OTE) & $\sqrt{ }$ & - & $\sqrt{ }$ & - \\
\hline \hline
\end{tabular}

\section{RESULTS AND DISCUSSION}

\section{A. Anomalous Green's functions}

The linearized Eilenberger equations allow us to study the direct crossover from the diffusive to the ballistic regime of quasiparticle transport, and hence dependence of the different 

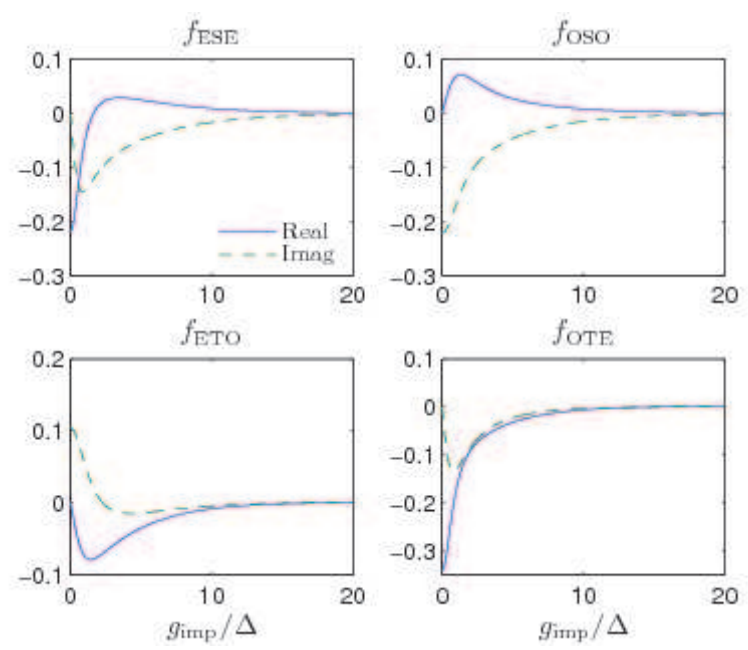

FIG. 2: (color online) Plot of the proximity-induced anomalous Green's functions in the middle of the ferromagnetic region $(x / d=$ $0.5)$ using $h / \Delta=15$ and $\varepsilon / \Delta_{0}=0.5$.

symmetry components on the impurity scattering. In the experimental situation, one usually probes the DOS at the F/I interface $x=d$, although it in principle is possible to obtain a spatially resolved DOS in the entire ferromagnetic region by using local scanning tunneling microscopy (STM)measurements. Let us first focus on $x=d$ and consider the ballistic limit in which case simple and transparent analytical expressions may be obtained from Eqs. (28). In the case $h \neq 0$, we obtain

$$
\begin{aligned}
& f_{\mathrm{ESE}}=\sum_{\sigma}[-2 \gamma s(\theta)] / \sinh \left(\lambda_{\sigma} d\right), f_{\mathrm{OSO}}=0, \\
& f_{\mathrm{ETO}}=0, f_{\mathrm{OTE}}=\sum_{\sigma}[-2 \sigma \gamma s(\theta)] / \sinh \left(\lambda_{\sigma} d\right)
\end{aligned}
$$

Note that for $h=0, \lambda_{\sigma}$ becomes independent of $\sigma$, leading to $f_{\mathrm{OTE}}=0$. At first glance, this appears to be in contradiction to Tab. [since the odd-parity components are absent even in the ballistic limit. However, evaluation of Eqs. (28) for $x \neq d$ reveals that these components are in general induced, as they should be. It is remarkable that the odd-parity components vanish exactly right at the $\mathrm{F} / \mathrm{I}$ interface. In the presence of a finite exchange field $h \neq 0$, however, the odd-frequency component $f_{\text {OTE }}$ survives at $x=d$, and its influence on physical quantities such as the DOS may be directly probed there. These results suggest that in order to investigate the influence of the odd-frequency superconducting correlations $f_{\mathrm{ETO}}$ and $f_{\text {Oso }}$, one would have to measure the DOS at several positions in the ferromagnetic region and not only at the $\mathrm{F} / \mathrm{I}$ interface. In Fig. 2, we plot the different symmetry components of the anomalous Green's function in the ferromagnet and their dependence on the impurity level.

\section{B. Density of states}

To demonstrate the applicability of Eq. (25), we study in particular how the DOS depends on the crossover from the ballistic $\left(g_{\text {imp }}=0\right)$ to the diffusive limit $\left(g_{\mathrm{imp}} \gg\right.$ $\left.\left\{h, \varepsilon, \Delta_{0} . g_{\mathrm{sf}}\right\}\right)$. We will fix $\gamma=0.05$ and $h / \Delta_{0}=15$ to model a realistic experiment, corresponding to a weak ferromagnetic alloy like $\mathrm{Cu}_{1-x} \mathrm{Ni}_{x}$ or $\mathrm{Pd}_{1-x} \mathrm{Ni}_{x}$. The setup is shown in Fig. 3, It is well-known that the DOS oscillates in space upon penetration deeper into the ferromagnetic region ${ }^{40}$ due to the presence of an exchange field, a feature which is robust both in the clean and dirty limit. However, the energydependence of the DOS in the presence of an arbitrary impurity concentration has not received much attention so far. This is because most works concerned themselves with the simplified Usadel equation (diffusive limit) or the Eilenberger equation in the absence of impurities (clean limit).

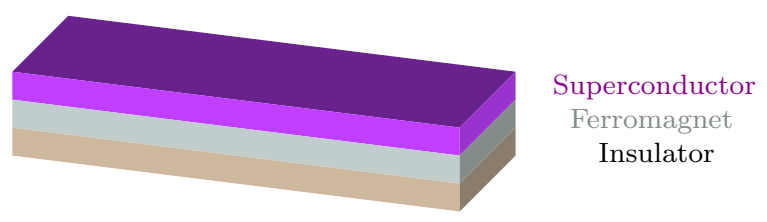

FIG. 3: (color online) Setup for our study of the density of states.

In Ref. 18, corrections to the normal-state DOS as induced by the proximity effect were calculated under the assumption that $h \tau_{\text {imp }} \gg 1$. This case corresponds to a ferromagnet where the exchange field is considerably larger than the self-energy associated with the impurity scattering. This may describe either a strong ferromagnet (one must still demand $h \ll \varepsilon_{F}$ ) or a weak ferromagnet with weak impurity scattering. Neither of these cases are possible to treat with the Usadel equation. In the present work, however, we do not impose any restrictions on the parameter $h \tau_{\text {imp }}$, which allows us to study the full crossover regime. This may be important in order to obtain a larger degree of consistency between theory and experimental data in the case when the diffusive limit is not fully reached.

In Fig. 4ha), we study the energy-resolved DOS for an intermediate range of impurity scattering. As a measure of the junction width, we use the superconducting coherence length in the clean limit $\xi_{S}=v_{F} / \Delta_{0}$. To isolate the role of the impurity scattering, we fix the junction width at $d / \xi_{S}=0.5$. For a superconductor with $v_{F}=10^{5} \mathrm{~m} / \mathrm{s}$ and $\Delta_{0}=1 \mathrm{meV}$, this corresponds to $d \simeq 30 \mathrm{~nm}$, which is experimentally relevant. As seen, the DOS exhibits a slightly oscillating behaviour as a function of energy when the impurity scattering rate $g_{\text {imp }}$ is comparable in magnitude to the superconducting gap. This effect becomes more obvious for wider junctions $d / \xi_{S} \gg 1$, and is attributed to bound states appearing in the ferromagnetic film. We discuss this in more detail below. As $g_{\text {imp }}$ increases, however, the DOS becomes featureless for subgap energies although one may still observe an alternating positive and negative correction to the zero-energy DOS upon increasing $g_{\text {imp }}$. In Fig. 4b), we plot the spatially-resolved DOS at $\varepsilon=0$ for various rates of the impurity scattering, in- 
cluding the case when $h \tau_{\text {imp }} \sim 1$. As seen, the oscillations of the zero-energy DOS are reduced with increasing impurity scattering. We have also investigated the effect of spin-flip scattering for an intermediate value of the impurity concentration. The spin-flip scattering, here taken to be uniaxial, is pair-breaking and thus suppresses the proximity-effect induced by the superconductor. This aspect agrees with Ref.41, which found that both the triplet and singlet components are suppressed with uniaxial and/or isotropic spin-flip scattering. For other types of magnetic scattering, such as planar spin-flip or spin-orbit scattering, the singlet and triplet components are affected very differently 11 .

The oscillations of the DOS in S/F junctions are usually attributed to the oscillating decay of the Cooper pair wave- function in the ferromagnetic region. In an $\mathrm{S} / \mathrm{N}$ junction, this decay is monotonous, and hence one would not expect to see any oscillations in the DOS. However, we underline that the impurity scattering plays an important role in this respect. In the ballistic case $g \rightarrow 0$, the proximity of the superconductor induces Andreev-bound states with well-defined trajectories which propagate in the normal part of the system. The statistical distribution of all possible trajectories is peaked at given lengths, typically at trajectories corresponding to the first and second reflection processes at the interface. As a result, the DOS in a clean $\mathrm{S} / \mathrm{N}$ junction acquires oscillations both as a function of energy and coordinate inside the normal region as seen in Fig. 5 upon averaging over all possible trajectories. This effect is known as Tomasch-oscillations ${ }^{42}$.
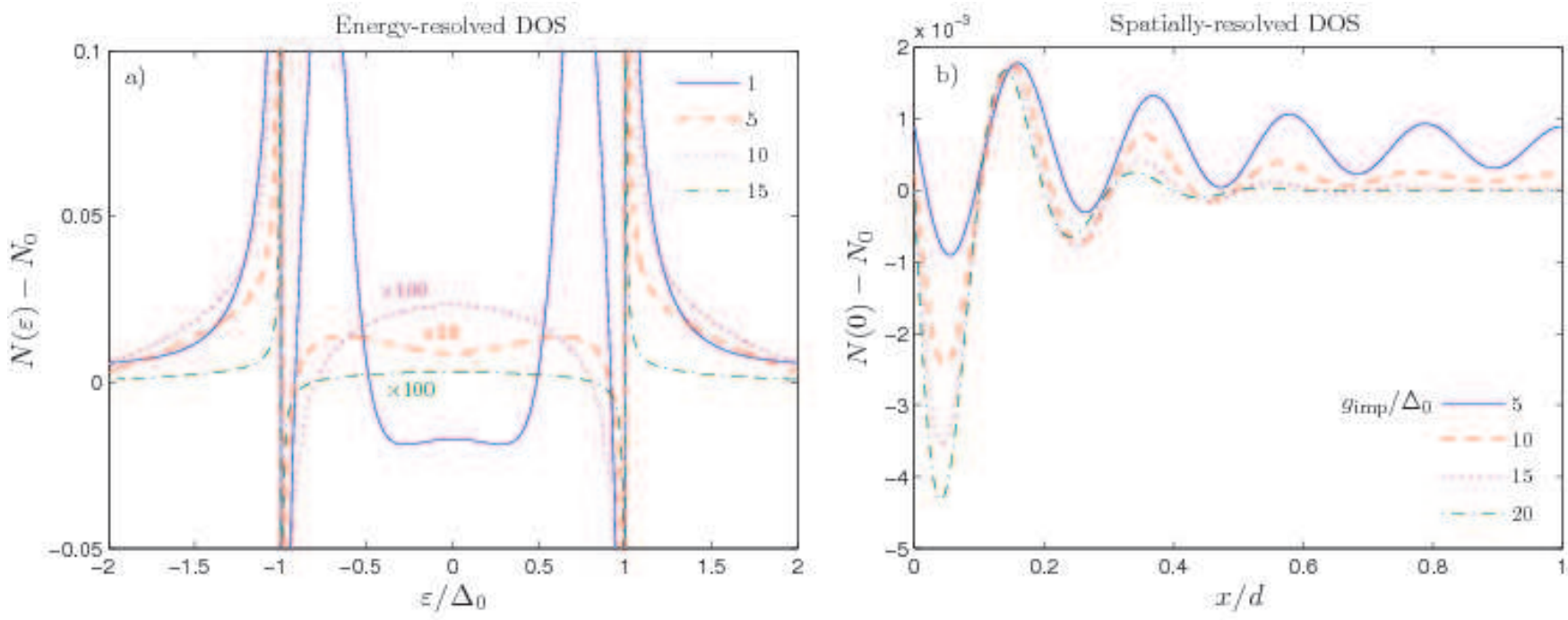

FIG. 4: (color online) Plot of the a) energy-resolved DOS at $x=d$ and b) spatially-resolved DOS at $\varepsilon=0$ for several values of the impurity scattering rate. Here, the exchange field is set to $h / \Delta_{0}=15$ and $d / \xi=0.5$.

However, there is another point which has appears to have been overlooked in the literature: namely that the spatial oscillations of the DOS in a $\mathrm{S} / \mathrm{N}$ junction at finite energies do not vanish in the diffusive limit. Hence, the oscillating DOS as a function of distance penetrated into the non-superconducting region is not a feature pertaining uniquely to $\mathrm{F} / \mathrm{S}$ junctions, as have been implied in some works ${ }^{43}$. To see this, we plot the spatially-resolved DOS both for a F/S and N/S junction in Fig. 6 in the diffusive regime. The curves are obtained by using the framework of Ref ${ }^{41}$, and thus correspond to a full numerical solution of the Usadel equation without restricting ourselves to the weak proximity effect regime. The oscillations of the DOS in the N/S case may be understood by noting that the induced superconducting Green's function in the normal region has a finite center-of-mass momentum $q=2 \varepsilon / v_{F}$. This is typically much smaller than the center-of-mass momentum acquired in a ferromagnet, $q=2 h / v_{F}$, which means that the corresponding oscillation length is much larger, but still present.

Having stated this, it should be noted that the oscillating nature of the anomalous Green's function does not necessarily imply that the critical temperature dependence or the Josephson current in N/S multilayers is non-monotonuous, e.g. displaying $0-\pi$ oscillations, since the energy-dependence of the Green's functions is integrated out when obtaining the critical temperature or critical current. For an F/S junction, on the 

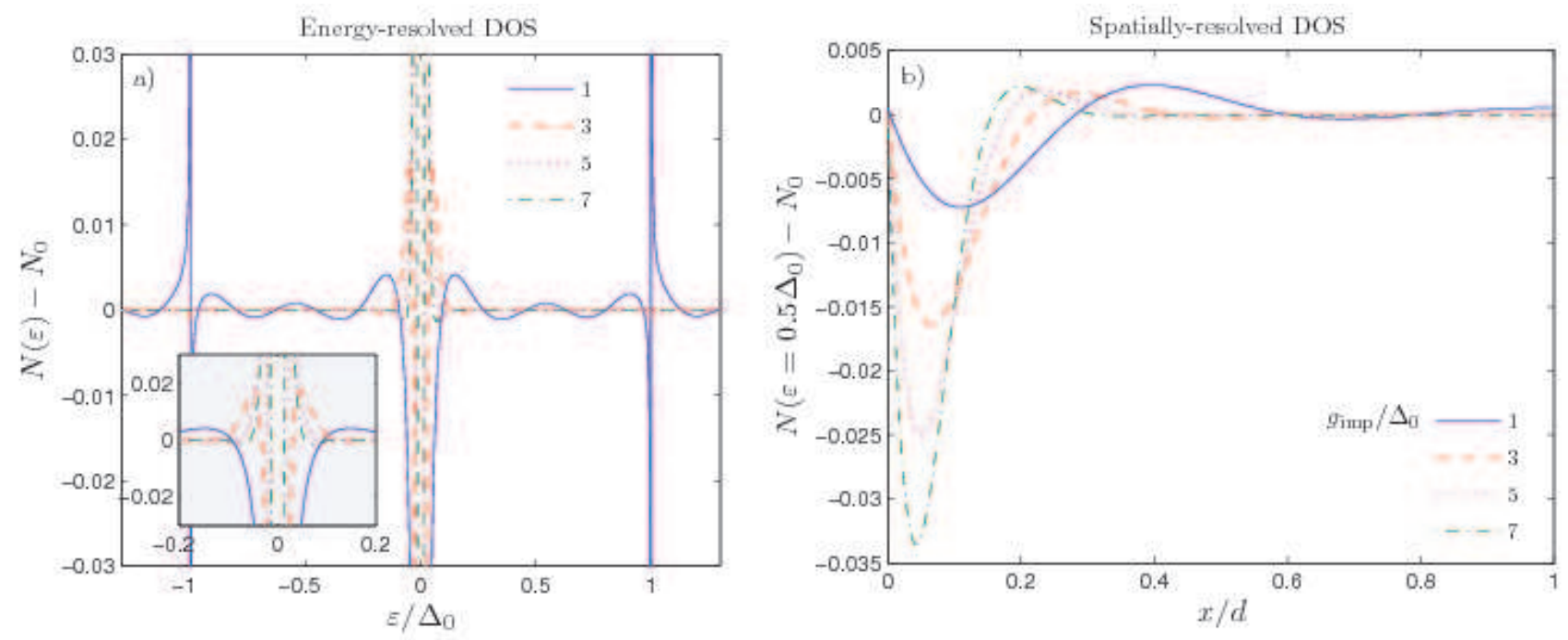

FIG. 5: (color online) Plot of the a) energy-resolved DOS at $x=d$ and b) spatially-resolved DOS at $\varepsilon / \Delta_{0}=0.5$ for several values of the impurity scattering rate. Here, the exchange field is set to zero, corresponding to a normal metal, and $d / \xi=5.0$.

other hand, the Cooper pair wave-function may retain its oscillating character even after the energy-integration since the center-of-mass momentum depends on the exchange field $h$.
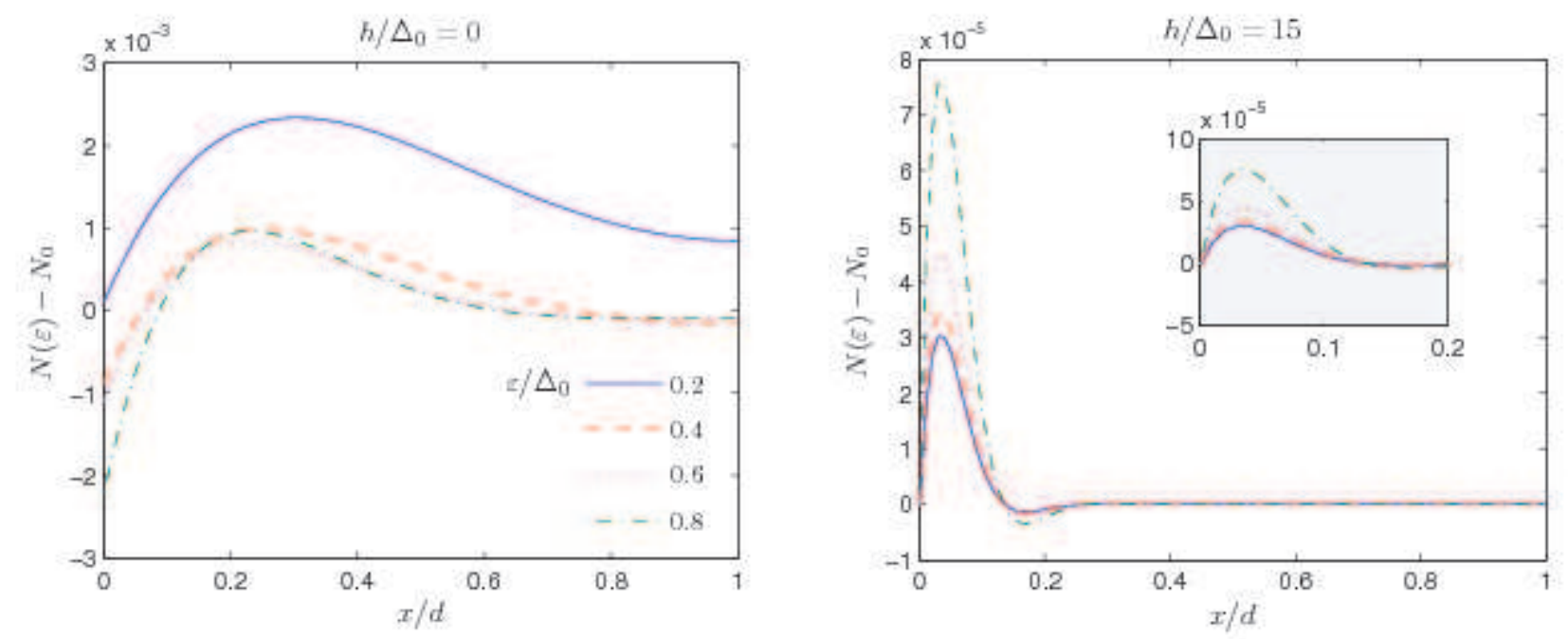

FIG. 6: (color online) Plot of the spatially resolved DOS for a diffusive N/S and F/S junction, respectively. In both cases, oscillations of the DOS are seen at finite energies. We have here fixed $d / \xi=3.0$ and $\tau=0.2$, using the notation of Ref. ${ }^{41}$ (here, $\xi=\sqrt{D / \Delta_{0}}$ while $\tau$ denotes the barrier transparency).

\section{Josephson current}

We now evaluate the Josephson current in an SFS junction for an arbitrary impurity concentration, with a setup as shown in Fig. 7. Denoting the phase at the left (right) superconductor as $+\chi(-\chi)$, the total phase difference is given by $\varphi=2 \chi$. 


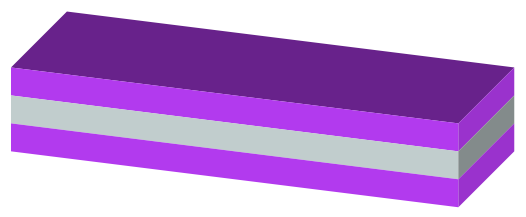

Superconductor Ferromagnet Superconductor

FIG. 7: (color online) Setup for our study of the Josephson current.

The current through the junction is evaluated by

$$
\mathbf{I}_{J}=\frac{N_{F} S_{0} e v_{F}}{4} \int \mathrm{d} \varepsilon \tanh (\beta \varepsilon / 2) \operatorname{Tr}\left\{\left\langle\hat{\rho}_{3} \mathbf{e}_{F}\left(\hat{g}^{R}-\hat{g}^{A}\right)\right\rangle\right\}
$$

under the assumption of equilibrium distribution functions. Here, $S_{0}$ is the effective area of the contact through which the current flows, while $\beta=1 / T$ is inverse temperature. Experimentally, one measures the current that flows through the junction, corresponding to the $x$-direction here. We employ the following boundary conditions:

$$
\begin{array}{r}
\left.\underline{\mathcal{A}_{\sigma, F}}\right|_{x=0}=\left.\gamma\left[\mathcal{S}_{\sigma, F}, \mathcal{S}_{\sigma, S}^{\text {Left }}\right]_{-}\right|_{x=0}, \\
\underline{\left.\mathcal{A}_{\sigma, F}\right|_{x=d}}=-\left.\gamma\left[\mathcal{S}_{\sigma, F}, \mathcal{S}_{\sigma, S}^{\text {Right }}\right]_{-}\right|_{x=d},
\end{array}
$$

and approximate $\mathcal{S}_{\sigma, F}=\underline{\tau_{3}}$ as in the previous section, in accordance with our assumption of a weak proximity effect. After some calculations, we arrive at the following expression for the Josephson current:

$$
\begin{aligned}
& I_{J}=4 \gamma^{2} N_{F} S_{0} e v_{F} I_{c} \sin \varphi, \text { with the definition } \\
& I_{c}=\int_{-\infty}^{\infty} \mathrm{d} \varepsilon \sum_{\sigma} \operatorname{Re}\left\{\frac{s^{2}(\theta)\left(1-G_{\sigma}\right) \tanh (\beta \varepsilon / 2)}{1\left(1+G_{\sigma}\right) \sinh \left(\lambda_{\sigma} d\right)}\right\} .
\end{aligned}
$$

The reader is reminded of the definitions

$$
\begin{aligned}
G_{\sigma} & =g /\left(\sqrt{r_{\sigma}^{2}-g^{2}}+r_{\sigma}\right), \\
r_{\sigma} & =21(\varepsilon+\sigma h)-\left(g_{\mathrm{imp}}+3 g_{\mathrm{sf}}\right) / 2, \\
g & =\left(g_{\mathrm{imp}}-g_{\mathrm{sf}}\right) / 2, g_{\mathrm{imp}(\mathrm{sf})} \equiv \tau_{\mathrm{imp}(\mathrm{sf})}^{-1} .
\end{aligned}
$$

Eq. (32) is the second of our three main analytical results in this work. It is probably the most compact way of expressing the Josephson current for arbitrary exchange fields and impurity scattering rates within the quasiclassical framework. It is thus suitable both for the case of a weak ferromagnet (such as the alloy $\mathrm{Cu}_{1-x} \mathrm{Ni}_{x}$ ), and for strong ferromagnets (like $\mathrm{Co}$ or $\mathrm{Fe}$ ) regardless of whether they are clean or dirty. In experiments performed with such strong ferromagnets, where the exchange field may be of order $100 \mathrm{meV}\left(\gg T_{c}\right)$, the Usadel equation is not valid at the same time as the clean limit may not be fully reached. In this case, one has to use an expression valid for the crossover regime, which emphasizes the importance of Eq. (32).

Below, we will study how impurity scattering affects both the width- and temperature-dependence of the critical current, as well as its belonging $0-\pi$ phase diagram. Bergeret $e t$ al. $\stackrel{19}{=}$ investigated this in the limiting cases of $h \tau_{\text {imp }} \ll 1$ and $h \tau_{\text {imp }} \gg 1$, while the majority of studies so far considered exclusively the limiting case of diffusive motion. We here pay particular attention to the crossover between the ballistic and diffusive sector, which has not been investigated previously. To model inelastic scattering, we add a small imaginary number to the quasiparticle energy, $\varepsilon \rightarrow \varepsilon+1 \delta$ where $\delta=10^{-3}$.

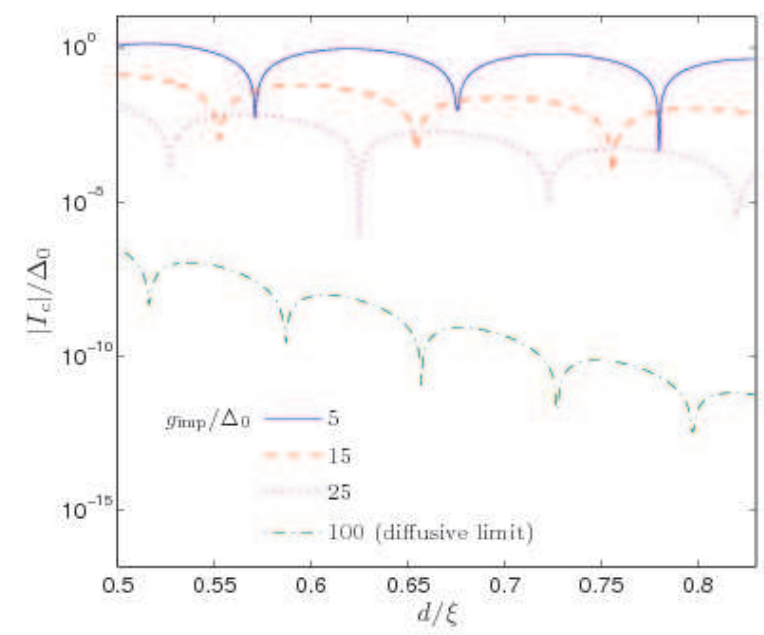

FIG. 8: (color online) Plot of the critical current as a function of junction width $d$. We have used $T / T_{c}=0.2$.

In Fig. 8, we plot the width-dependence of the critical current for a temperature $T / T_{c}=0.2$. As seen, increasing impurity scattering suppresses the magnitude of the current and also reduces the oscillation length $l_{\text {osc }}$. The dependence of the latter on impurity scattering is shown explicitly in Fig. 9 . Using the Usadel equation, it is predicted that the oscillation length of the critical current in the dirty limit should depend on the impurity scattering rate like $\sqrt{h l_{\text {imp }}} \sim \sqrt{\tau_{\text {imp }}}$ (for a discussion of the characteristic decay and oscillation lengths in the clean and dirty limit, see Tab. I in Ref $\stackrel{2}{=}$ ). We obtain a good fit with this in Fig. 9 when $g_{\text {imp }} \gg \Delta$. For values of $g_{\text {imp }}$ comparable to $\Delta$, however, the oscillation length saturates at a finite value. In the ballistic limit, the oscillation length is known to depend on the exchange field like $1 / h$. We have also confirmed this for several values of $h$ when $g_{\text {imp }} \sim \Delta$.

Also, one notes from Fig. 8 that the decay length of the current increases with the concentration of impurities. It should be noted that the measure $\xi$ used as a length unit in this context is independent of the impurity scattering rate, since we are using $\xi=v_{F} / \Delta_{0}$. This way, we ensure that the effects observed are really due to the increased impurity scattering. If we for instance had used the mean free path $l_{\mathrm{mfp}}=v_{F} \tau_{\mathrm{imp}}$ as a measure for the junction width, the scale would have been different for each value of $g_{\text {imp }}$ in Fig. 8. We also underline that the dirty limit condition is that $\xi / l_{\mathrm{mfp}} \gg 1$, while the size $d$ of the sample may be either smaller or larger than $\xi$ as long as that condition is fulfilled.

In Fig. 10, we pay particular attention to the case $h \tau_{\text {imp }}=1$ which is inaccessible in the Usadel framework. As seen, nothing qualitatively new shows up in the $d$-dependence or the $T$ - 


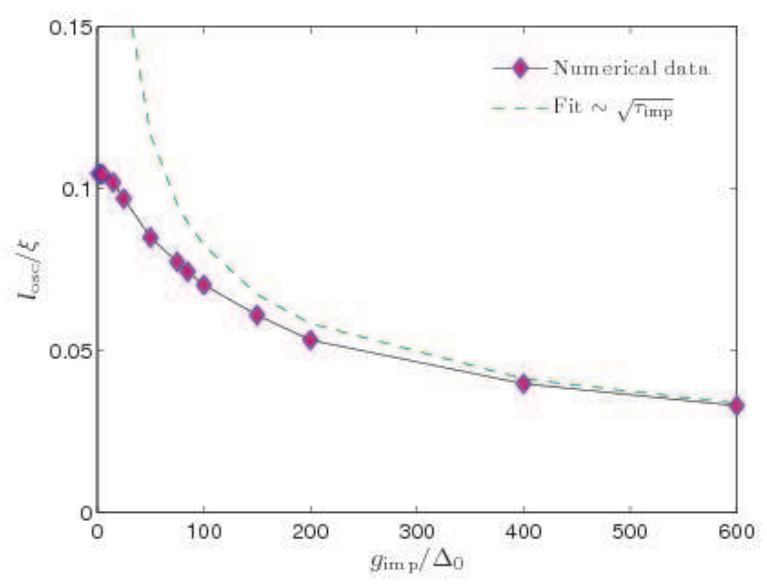

FIG. 9: (color online) Plot of the oscillation length of the critical current as a function of the impurity scattering strength $g_{\mathrm{imp}}$. We have used $T / T_{c}=0.2$.

dependence of the critical current as compared to the diffusive limit, although the decay rate is considerably lower. We also investigate how the $0-\pi$ phase diagram of the Josephson junction is affected by impurity scattering. This is most conveniently plotted in the $d-T$ plane. In Fig. 11, one observes several features. First, it is clear that the area occupied by the 0 and $\pi$ phases, respectively, diminishes with increasing $g_{\text {imp }}$, in agreement with the shortened oscillation length of Fig. 9. Secondly, it is seen that thermal $0-\pi$ transitions are practically speaking impossible to observe for scattering rates satisfying $g_{\text {imp }} \leq h$. As the scattering rate is increased, however, the thermal transitions become possible when $g_{\text {imp }} \gg h$, or equivalently $h \tau_{\text {imp }} \ll 1$. In this regime, the Usadel equation is valid and we obtain consistency with previous results. At all scattering rates, the width-induced transitions are possible.

\section{Critical temperature}

Finally, we investigate an $\mathrm{F} / \mathrm{S} / \mathrm{F}$ layers where the critical temperature of the superconductor is sensitive to the relative orientation of magnetization of the two F layers. This effect is usually dubbed to a spin-switch effect in the literature. Our setup is shown in Fig. 12 Tagirov $\underline{44}$ was the first to point out the interesting opportunity to "activate" superconductivity simply by means of switching the direction of the magnetization in one of the ferromagnetic layers. Since then, a number of works have elaborated on the spin-switch effect both experimentally $\frac{45,46,47}{4}$ and theoretically ${ }^{40,48,49,50}$. In particular, a convincing numerical approach was developed in Ref ${ }^{49}$. So far, however, almost all theoretical works focused on the dirty limit, in which the critical temperature may be conveniently calculated by using the Usadel equation in the Matsubara frequency representation. Although the obtained results compare well qualitatively with experimental data, an unsolved factor so far is the discrepancy of two orders in magnitude of the
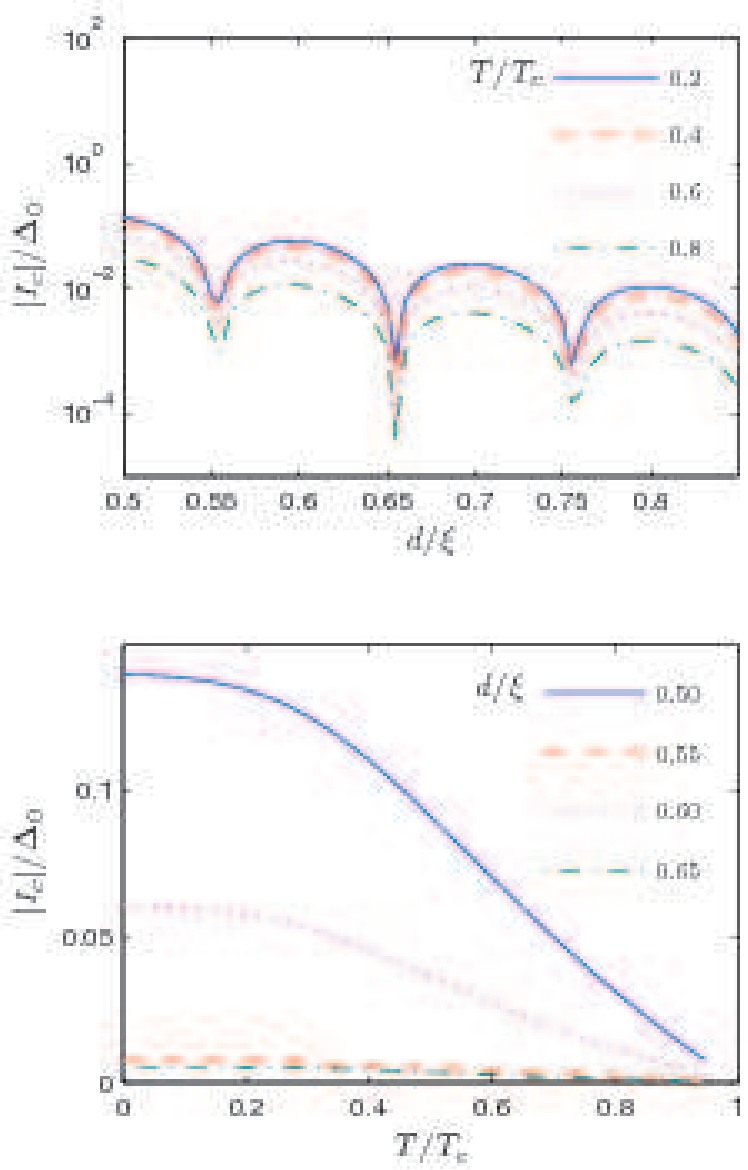

FIG. 10: (color online) Plot of the $d$-dependence and the $T$ dependence of the critical current for $g_{\text {imp }} / \Delta_{0}=15$, corresponding to $h \tau_{\text {imp }}=1$.

predicted effect. Recently, it was proposed and investigated ${ }^{51}$ if an asymmetry in the interface transparencies of the F/S/F junctions could be responsible for this, in effect one of the interfaces was much less transparent than the other. The authors of Ref $\frac{51}{1}$ concluded that this was not the case. At present, the single ferromagnet $\mathrm{F} / \mathrm{S} / \mathrm{F}$ devices to have been examined so far have used strong ferromagnets, which falls outside the range of applicability of the Usadel equation ${ }^{45,46,47}$. In light of this, it would be interesting to go beyond the usual treatment with the Usadel equation and solve the more general Eilenberger equation to investigate the role of the impurity scattering.

A general analytical solution for arbitrary proximity effect and barrier transparency is hardly achievable, as pointed out previously. Nevertheless, it is reasonable to expect that one may capture the essential physics in the weak proximity effect regime. In order to calculate the critical temperature for the $\mathrm{P}$ and AP alignment, we assume that the temperature is close to $T_{c}$, which allows us to write the Green's function in the 

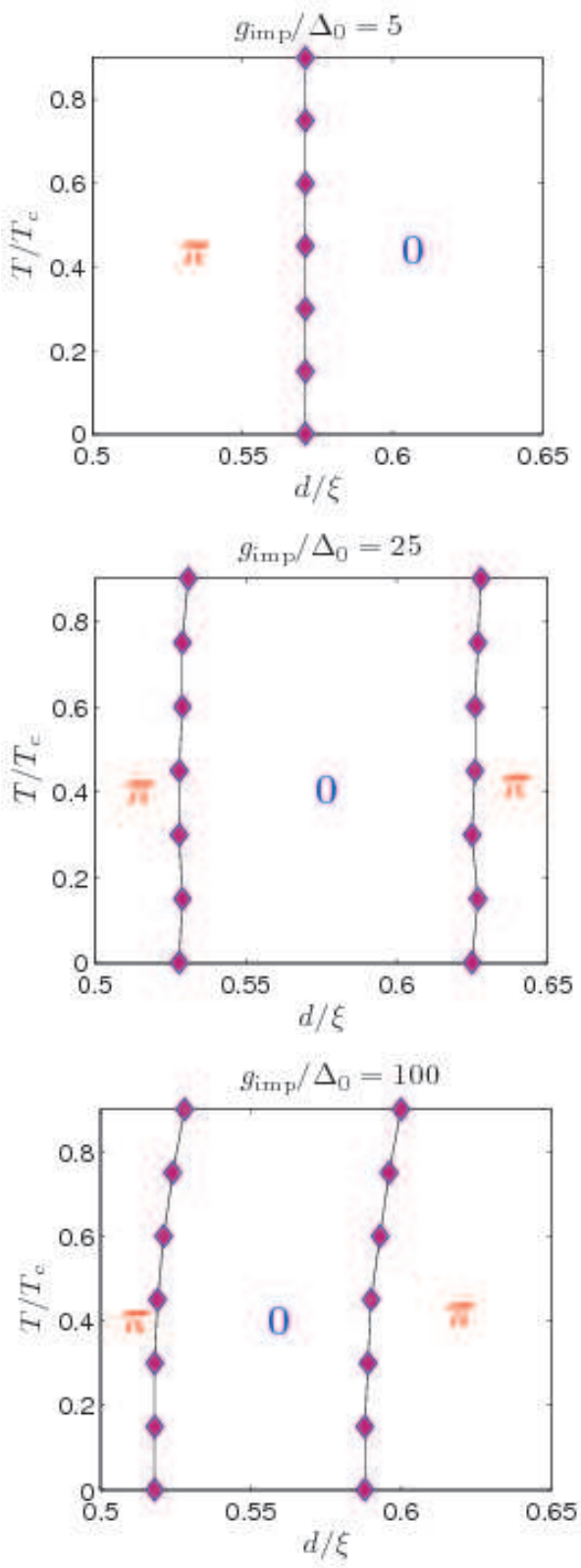

FIG. 11: (color online) Phase diagram in the $d-T$ plane for the $0-\pi$ transitions of the critical current for several values of the impurity concentration. superconductor as follows:

$$
\underline{g_{\sigma}}=\left(\begin{array}{rr}
1 & 2 a_{\sigma} \\
2 b_{\sigma} & -1
\end{array}\right)
$$

since $\lim _{\Delta \rightarrow 0} c(\theta)=1$. For the normal part of the Green's function matrix, this means that $\left(1-a_{\sigma} b_{\sigma}\right) /\left(1+a_{\sigma} b_{\sigma}\right) \simeq$ 1 , while for the anomalous Green's function one thus has $2 a_{\sigma} /\left(1+a_{\sigma} b_{\sigma}\right) \simeq 2 a_{\sigma}$. The self-consistency equation for the superconducting gap reads in general ${ }^{52}$

$$
\Delta=\frac{N_{F} \lambda}{8} \operatorname{Tr}\left\{\left(\frac{\hat{\rho}_{1}-1 \hat{\rho}_{2}}{2}\right) \hat{\tau}_{3} \int \mathrm{d} \varepsilon\left\langle\hat{g}^{K}\right\rangle\right\}, \lambda>0,
$$

where $\lambda$ is the attractive interaction and $\hat{g}^{K}$ is the Keldysh part of the Green's function. For an equilibrium situation $\left[\hat{g}^{\mathrm{K}}=\right.$ $\left.\left(\hat{g}^{\mathrm{R}}-\hat{g}^{\mathrm{A}}\right) \tanh (\beta \varepsilon / 2)\right]$ in the weak-proximity effect regime with a temperature very close to $T_{c}$, this reduces to

$$
\Delta=\frac{N_{F} \lambda}{8} \int \mathrm{d} \varepsilon \tanh \left(\frac{\varepsilon}{2 T_{c}}\right) \sum_{ \pm} \sum_{\sigma} \sigma\left[a_{\sigma}^{ \pm}-\left(b_{\sigma}^{ \pm}\right)^{*}\right]
$$

Once the anomalous Green's functions $\left\{a_{\sigma}^{ \pm}, b_{\sigma}^{ \pm}\right\}$have been obtained, one may solve Eq. (37) numerically to obtain $T_{c}$ in the $\mathrm{P}$ and AP configurations. Using boundary conditions explained below, we solve for the anomalous Green's functions in both the ferromagnetic and superconducting regions and obtain the following equation determining the critical temperature:

$$
1-N_{F} \lambda \int_{0}^{\omega} \mathrm{d} \varepsilon \tanh \left(\frac{\varepsilon}{2 T_{c}}\right) \varepsilon^{-1}\left[1-\cos \left(2 \varepsilon x / v_{F}\right)-\sum_{\sigma, \pm} \operatorname{Re}\left\{\frac{L_{\sigma}^{ \pm} \mathrm{e}^{ \pm 21 \varepsilon x / v_{F}} \sum_{\alpha} \alpha R_{\sigma}^{\alpha}\left(1-\mathrm{e}^{2 \alpha 1 \varepsilon d_{S} / v_{F}}\right)}{4 \sum_{\alpha} \alpha \mathrm{e}^{2 \alpha 1 \varepsilon d_{S} / v_{F}} L_{\sigma}^{\alpha} R_{\sigma}^{\alpha}}\right\}\right]=0
$$

with the cut-off energy $\omega, \alpha= \pm$, and finally

$$
\begin{aligned}
& L_{\sigma}^{ \pm}=\mathrm{e}^{ \pm \lambda_{\sigma}^{\text {Left }} d_{F}}-G_{\sigma}^{\text {Left }} \mathrm{e}^{\mp \lambda_{\sigma}^{\text {Left }} d_{F}}, \\
& R_{\sigma}^{ \pm}=\mathrm{e}^{ \pm \lambda_{\sigma}^{\text {Right }} d_{F}}-G_{\sigma}^{\text {Right }} \mathrm{e}^{\mp \lambda_{\sigma}^{\text {Right }} d_{F}} .
\end{aligned}
$$

Eq. (37) is the third of our three main analytical results in this work. It gives an expression for the critical temperature in an $\mathrm{F} / \mathrm{S} / \mathrm{F}$ junction for arbitrary exchange fields and impurity 
scattering rates within the framework of quasiclassical theory in the weak-proximity effect regime.

In order to find $\left\{a_{\sigma}^{ \pm}, b_{\sigma}^{ \pm}\right\}$, we must introduce proper boundary conditions at each of the interfaces in the setup (Fig. 12). The left ferromagnet is assumed to occupy the region $-d_{F}<$ $x<0$, the superconductor is located at $0<x<d_{S}$, while the right ferromagnet occupies the space $d_{S}<x<d_{S}+d_{F}$. Thus, the ferromagnetic layers are assumed to have the same thickness $d_{F}$ while the superconductor has thickness $d_{S}$. Due to the complexity of the problem, we will assume rigid boundary conditions at the superconductor/ferromagnet interfaces, which amounts to continuity of the Green's function. Although the low transparency limit is probably more realistic, it is reasonable to expect qualitatively correct results in this approach. Moreover, since we already assume a temperature close to $T_{c}$, the proximity effect would be almost completely absent if we in addition incorporated tunneling interfaces. In general, high transparency interfaces cause a depletion of the superconducting order parameter near the interface, which means that one should strictly speaking solve for the spatial depletion of the gap self-consistently. In our approach, we do not incorporate this depletion since we are aiming for analytical results. A full numerical approach would, however, doubtlessly improve the accuracy of the results presented below, but at the prize of losing the analytical information.

At the ends of the ferromagnetic layers, we impose vacuum boundary conditions. In total, the boundary conditions then read:

$$
\begin{aligned}
x=-d_{F}: & \underline{\mathcal{A}_{\sigma, F}^{\text {Left }}=\underline{0},} \\
x=0: & \underline{g_{\sigma, F}^{\text {Left }}}=\underline{g_{\sigma}}, \\
x=d_{S}: & \underline{g_{\sigma}}=g_{\sigma, F}^{\text {Right }}, \\
x=d_{S}+d_{F}: & \underline{\mathcal{A}_{\sigma, F}^{\text {Right }}=\underline{0},}
\end{aligned}
$$

After straight-forward calculations, we obtain an expression

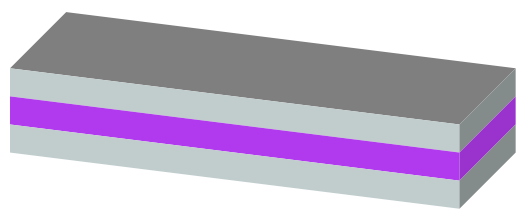

Ferromagnet

Superconductor Ferromagnet

With the analytical solution in hand, we now present a study of the critical temperature in the $\mathrm{P}$ and AP configuration, investigating in particular the role of impurity scattering. First, we plot the critical temperature as a function of ferromagnetic layer thickness with a fixed superconducting layer thickness of $d_{S} / \xi=0.03$ in Fig. 13. Using a superconductor with $\xi=200 \mathrm{~nm}$, this would correspond to a thickness $d_{S}=6$ $\mathrm{nm}$. To ensure the validity of our assumption that the anomalous Green's functions vary little with $x$ throughout the su-
FIG. 12: (color online) Setup for our study of the critical temperature.

for $\left\{a_{\sigma}^{ \pm}, b_{\sigma}^{ \pm}\right\}$in the superconductor. A few comments with regard to the expression Eq. (37) are in order. Firstly, it should be noted that the expression for the critical temperature in Eq. (37) depends on the position $x$ in the superconductor through the spatial dependence of the anomalous Green's function. This dependence is of course artificial and a result of the approximations we have made in the calculations; in a real experimental sample, $T_{c}$ is a property for the entire layer and does not depend on the position in the superconductor. The reason for why we obtain an artificial $x$-dependence in the expression for the critical temperature is because we have neglected the spatial modification of the order parameter $\Delta$ in the layer. Employing a fully self-consistent calculation would remove the spatial dependence of $T_{c}$ in the gap equation. However, for thin superconducting layers $d_{S} / \xi \ll 1$, our approximation is expected to be good. A similar procedure has been used in several other works which calculated $T_{c}$ by means of the Usadel equation. In those works, it was assumed that the anomalous Green's function in the superconductor varied very little as long as $d_{S} / \xi \ll 1$ was satisfied, and hence one could ignore the spatial dependence of the Green's function once it had been found. More precisely, $T_{c}$ was evaluated in the middle of the superconducting region. In our case, we will use the same approximation since our approach is analytical in nature. The main contribution to the integral in Eq. (37) comes from energies $\varepsilon \leq \Delta$, for which the terms including the coordinate $x$ on the right hand side of the equation change very little as long as $d_{S} / \xi \ll 1$. We will focus on the difference between the critical temperature in the $\mathrm{P}$ and $\mathrm{AP}$ alignments, defined as

$$
\Delta T_{c} \equiv T_{c}^{\mathrm{AP}}-T_{c}^{\mathrm{P}}
$$

We will normalize all temperatures on $T_{c}^{0}$, which is the bulk critical temperature of the superconductor in the absence of a proximity effect. As demanded by consistency, the critical temperature approaches $T_{c}^{0}$ when $d_{F} \rightarrow 0$. We choose the cut-off frequency as $\omega / \Delta_{0}=30$.

perconducting layer, we plot the critical temperature both at $x / d_{S}=0.50$ (symbols) and $x / d_{S}=0.01$ (dashed lines). As seen, the difference is neglible. From Fig. 13, one may infer that the critical temperature in the $\mathrm{P}$ configuration goes to zero much faster than in the AP configuration as a function of the ferromagnetic layer thickness $d_{F}$. This supports the notion that the antiparallell configuration favors superconductivity in the middle layer. The effect of impurity scattering is seen to suppress the critical temperature, in general. 

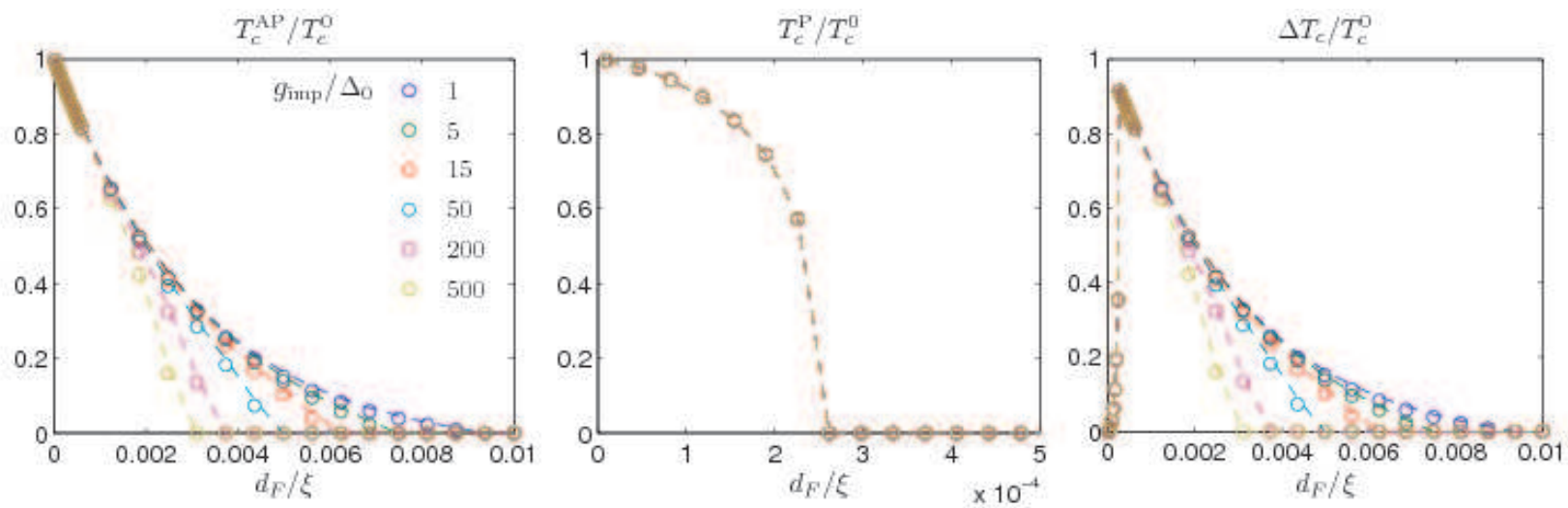

FIG. 13: (color online) Plot of the critical temperature in an F/S/F structure as a function of the ferromagnetic layer thickness $d_{F}$ for fixed $d_{S} / \xi=0.03$. Note the different scale for $d_{F}$ in the middle panel. The symbols denote the result for $x / d_{S}=0.50$ while the dashed lines correspond to $x / d_{S}=0.01$.

One may understand intuitively why the antiparallell alignment is favorable compared to the parallell alignment, since the average exchange field cancels in the former case. Qualitatively, our results are consistent with the monotonic decay found for a high barrier transparency when using the Usadel equation ${ }^{2}$. However, a more realistic scenario would clearly be to invoke low barrier transparency boundary conditions at the $\mathrm{S} / \mathrm{F}$ interfaces. Due to the complexity of the problem upon including an arbitrary amount of impurities, we have used perfectly transparent interfaces here as a first approximation. It would nevertheless be quite interesting to extend this formalism to low transparency interfaces to investigate the role of impurity scattering under those circumstances. Especially, the role of $g_{\text {imp }}$ with regard to the re-entrant behavior of $T_{c}$ would be worth investigating. Our analytical results may serve as a basis for extending this formalism to low transparency interfaces in the case of an arbitrary value for $h \tau_{\text {imp }}$, as opposed to $h \tau_{\text {imp }}$ in the Usadel regime.

\section{SUMMARY}

We have investigated various aspects of the physics resulting from the proximity effect in ferromagnet/superconductor $(\mathrm{F} / \mathrm{S})$ bilayers. In contrast to previous works, which were limited to either the clean or dirty limit, we have taken into account an arbitrary scattering rate for both non-magnetic and magnetic impurities. This has allowed us to access the crossover regime from the ballistic to diffusive regime of the proximity effect. We have derived analytical formula for $i$ ) the proximity-induced DOS of an F/S bilayer, ii) the Josephson current in an S/F/S junction, and iii) the critical temperature of an $\mathrm{F} / \mathrm{S} / \mathrm{F}$ structure. Our results are valid for an arbitrary ratio of the parameter $h \tau_{\mathrm{imp}}$, and are thus applicable both to weak ferromagnetic alloys as well as permalloys in either the diffusive or clean limit.

\section{Acknowledgments}

J. L. acknowledges T. Yokoyama, A. Cottet, F. S. Bergeret, and Ya. Fominov for useful discussions. H. Skadsem and M. Thaule are also thanked for valuable input. J.L. and A.S. were supported by the Norwegian Research Council Grant Nos. 158518/431, 158547/431, (NANOMAT), and 167498/V30 (STORFORSK). M. Z. thanks A. Brataas and A. Sudb $\varnothing$ for their hospitality and support during his visit to the Centre for Advanced Study, Oslo.

\section{APPENDIX}

The Pauli-matrices used in this paper are defined as

$$
\begin{aligned}
\underline{\tau_{1}} & =\left(\begin{array}{ll}
0 & 1 \\
1 & 0
\end{array}\right), \underline{\tau_{2}}=\left(\begin{array}{cc}
0 & -1 \\
1 & 0
\end{array}\right), \underline{\tau_{3}}=\left(\begin{array}{cc}
1 & 0 \\
0 & -1
\end{array}\right), \\
\underline{1} & =\left(\begin{array}{ll}
1 & 0 \\
0 & 1
\end{array}\right), \hat{1}=\left(\begin{array}{ll}
\underline{1} & \underline{0} \\
\underline{0} & \underline{1}
\end{array}\right), \hat{\tau}_{i}=\left(\begin{array}{ll}
\underline{\tau_{i}} & \underline{0} \\
\underline{0} & \underline{\tau_{i}}
\end{array}\right), \\
\hat{\rho}_{1} & =\left(\begin{array}{ll}
\underline{0} & \underline{\tau_{1}} \\
\underline{\tau_{1}} & \underline{0}
\end{array}\right), \hat{\rho}_{2}=\left(\begin{array}{cc}
\underline{0} & -\underline{1} \underline{\tau_{1}} \\
\underline{1} \underline{\tau_{1}} & \underline{0}
\end{array}\right), \hat{\rho}_{3}=\left(\begin{array}{cc}
\underline{1} & \underline{0} \\
\underline{0} & -\underline{1}
\end{array}\right) .
\end{aligned}
$$

${ }^{1}$ F. S. Bergeret, A. F. Volkov, and K. B. Efetov, Rev. Mod. Phys. 77, 1321 (2005).

2 A. I. Buzdin, Rev. Mod. Phys. 77, 935 (2005).

3 Yu.A. Izyumov, Yu.N. Proshin, M.G. Khusainov, PhysicsUspekhi 45, 109 (2002)
${ }^{4}$ M. L. Kulic and I. M. Kulic, Phys. Rev. B 63, 104503 (2001).

5 F. S. Bergeret, A. F. Volkov, and K. B. Efetov, Phys. Rev. Lett. 86, 3140 (2001).

${ }^{6}$ I. Eremin, F. S. Nogueira, and R.-J. Tarento, Phys. Rev. B 73, 054507 (2006). 
${ }^{7}$ Y. Zhao and R. Shen, Phys. Rev. B 73, 214511 (2006).

${ }^{8}$ M. S. Grønsleth, J. Linder, J.-M. Børven, and A. Sudbø, Phys. Rev. Lett. 97, 147002 (2006); J. Linder, M. S. Grønsleth, A. Sudbø, Phys. Rev. B 75, 024508 (2007).

9 Y. Asano, Phys. Rev. B 74, 220501 (2006).

${ }^{10}$ P. M. Brydon, Boris Kastening, D. K. Morr, and D. Manske, Phys. Rev. B 77, 104504 (2008).

11 T. Champel, T. Löfwander, and M. Eschrig, Phys. Rev. Lett. 100, 077003 (2008).

12 A. Brataas and Y. Tserkovnyak, Phys. Rev. Lett. 93, 087201 (2004).

13 D. Huertas-Hernando, Yu. V. Nazarov, and W. Belzig, Phys. Rev. Lett. 88, 047003 (2002).

14 F. Giazotto and F. Taddei, Phys. Rev. B 77, 132501 (2008).

15 J. Linder, T. Yokoyama, Y. Tanaka, and A. Sudbø, Phys. Rev. B 78, 014516 (2008).

16 K. Usadel, Phys. Rev. Lett. 25, 507 (1970).

17 G. Eilenberger, Sov. Phys. JETP 214, 195 (1968).

${ }^{18}$ F. S. Bergeret, A. F. Volkov, and K. B. Efetov, Phys. Rev. B 65, 134505 (2002).

${ }^{19}$ F. S. Bergeret, A. F. Volkov, and K. B. Efetov, Phys. Rev. B 64, 134506 (2001).

${ }^{20}$ M. Zareyan, W. Belzig, and Yu. V. Nazarov, Phys. Rev. B 65, 184505 (2002); M. Zareyan, W. Belzig, and Yu. V. Nazarov, Phys. Rev. Lett. 86, 308 (2001).

21 J. Kopu, M. Eschrig, J. C. Cuevas, and M. Fogelström, Phys. Rev. B 69, 094501 (2004).

${ }^{22}$ K. Halterman, O. T. Valls, and P. H. Barsic, Phys. Rev. B 77, 174511 (2008); K. Halterman, P. H. Barsic, and O. T. Valls, Phys. Rev. Lett. 99, 127002 (2007); K. Halterman and O. T. Valls, Phys. Rev. B 66, 224516 (2002).

23 J. Linder and A. Sudbø, Phys. Rev. B 75, 134509 (2007).

24 A. I. Buzdin, L. N. Bulaevskii and S. V. Panyukov, JETP Lett. 35, 178 (1982) [Pis'ma Zh. Eksp. Teor. Fiz. 35, 147 (1982)].

25 M. Fogelström, Phys. Rev. B 62, 11812 (2000)

${ }^{26}$ N. M. Chtchelkatchev, W. Belzig, Yu.V. Nazarov, and C. Bruder, JETP. Lett. 74, 323 (2001).

27 I. V. Bobkova and Yu. S. Barash, Phys. Rev. B 71, 144510 (2005)

28 P. SanGiorgio, S. Reymond, M. R. Beasley, J. H. Kwon, and K. Char, Phys. Rev. Lett. 100, 237002 (2008).

${ }^{29}$ N. Schopohl and K. Maki, Phys. Rev. B 52, 490 (1995); N. Schopohl, cond-mat/9804064.

30 A. V. Zaitsev, Sov. Phys. JETP 59, 1163 (1984).
31 C.-R. Hu, Phys. Rev. Lett. 72, 1526 (1994); J. Yang and C.-R. Hu, Phys. Rev. B 50, 16766 (1994).

32 T. Kontos, M. Aprili, J. Lesueur, and X. Grison, Phys. Rev. Lett. 86, 304 (2001).

33 Y. Tanaka et al. , Phys. Rev. Lett. 99, 037005 (2007).

${ }^{34}$ T. Yokoyama, Y. Tanaka, and A. A. Golubov, Phys. Rev. B 78, 012508 (2008).

35 V. L. Berezinskii, JETP Lett. 20, 287 (1974).

${ }^{36}$ E. Abrahams, A. Balatsky, D. J. Scalapino, and J. R. Schrieffer, Phys. Rev. B 52, 1271 (1995).

37 J. Linder, T. Yokoyama, and A. Sudbø, Phys. Rev. B 77, 174507 (2008).

38 Ya. V. Fominov, JETP Lett. 86, 732 (2007)

39 J. Linder, T. Yokoyama, Y. Tanaka, Y. Asano, and A. Sudbø, Phys. Rev. B 77, 174505 (2008).

${ }^{40}$ I. Baladie and A. Buzdin, Phys. Rev. B 64, 224514 (2001).

41 J. Linder, T. Yokoyama, and A. Sudbø, Phys. Rev. B 77, 174514 (2008).

${ }^{42}$ W. J. Tomasch, Phys. Rev. Lett. 15, 672 (1965).

43 A. Buzdin, Phys. Rev. B 62, 11377 (2000).

${ }^{44}$ L. R. Tagirov, Physica C 307, 145 (1998); L. R. Tagirov, Phys. Rev. Lett. 83, 2058 (1999).

45 A. Yu. Rusanov, S. Habraken, and J. Aarts, Phys. Rev. B 73, 060505(R) (2006).

46 I. C. Moraru, W. P. Pratt, Jr., and N. O. Birge, Phys. Rev. Lett. 96, 037004 (2006)

47 I. C. Moraru, W. P. Pratt, Jr., and N. O. Birge, Phys. Rev. B 74, 220507(R) (2006).

48 A. I. Buzdin, A. V. Vedyayev and N. V. Ryzhanova, Europhys. Lett. 48, 686 (1999).

49 Ya. V. Fominov, N. M. Chtchelkatchev, and A. A. Golubov, Phys. Rev. B 66, 014507 (2002).

50 T. Löfwander, T. Champel, and M. Eschrig, Phys. Rev. B 75, 014512 (2007).

51 P. Cadden-Zimansky, Ya. B. Bazaliy, L. M. Litvak, J. S. Jiang, J. Pearson, J. Y. Gu, Chun-Yeol You, M. R. Beasley, and S. D. Bader, Phys. Rev. B 77, 184501 (2008).

${ }^{52}$ We here notations and conventions close to the ones of J. P. Morten, M. Sc. thesis, Norwegian University of Science and Technology, 2003, which in turn are similar to J. W. Serene and D. Rainer, Phys. Rep. 101, 221 (1983). 